\title{
Canceling updating in the comprehension of counterfactuals embedded in narratives
}

\author{
Manuel de Vega and Mabel Urrutia \\ University of La Laguna, La Laguna, Canary Islands, Spain \\ AND \\ BERNARDO RIFFO \\ University of Concepción, Concepción, Chile
}

\begin{abstract}
Participants were given counterfactual sentences - for example, "If Mary had won the lottery she would have bought a Mercedes car" - or factual sentences - for example, "Because Mary won the lottery, she bought a Mercedes car" - embedded in short narratives. Reading times showed that readers were immediately sensitive to the special status of counterfactual information (Experiment 1). In addition, probe-recognition latencies demonstrated that old information was more accessible in counterfactual than in factual stories, and new information was equally accessible in both kinds of stories (Experiment 2). However, after reading additional clauses, new information became less accessible in counterfactual than in factual stories (Experiment 3 ). These results suggest that counterfactual events are momentarily represented but are later suppressed and the readers' attention goes back to previous events in the story.
\end{abstract}

We use language to describe facts: namely, what happened, is happening, or could happen. But we are also very skillful at producing and understanding counterfactual sentences such as "If Mary had won the lottery, she would have bought a Mercedes car." Counterfactual meaning has an unreal status, because it refers to a past event that did not happen and to the equally unreal consequence of such event. Counterfactuals might seem a pointless activity: Why do we expend our time and mental resources using expressions that do not describe real events or even possible future events? However, counterfactuals are a pervasive aspect of human mental life. The reasoning and social psychology literature provides wide evidence of the psychological functions of counterfactual thinking (see Byrne, 2002, 2005; Roese, 1997, 2005). Counterfactuals allow us to learn from mistakes; for instance, "If I had prepared for the exam I would have gotten an A score" may persuade the speaker to prepare better for the exam next time (Roese, 1994). By contrasting the outcome of an event with its counterfactual alternatives, people can work out causal judgments (Harris, German, \& Mills, 1996). Thus the counterfactual "If only John had been so kind as to bring me to the airport, I would have made it to my plane on time" allows the speaker to explain why she has missed her plane, attributing the responsibility to John's lack of kindness. In the same vein, counterfactual simulations of alternatives could be generated to predict the probability of an event (Kahneman \& Tversky, 1982). Finally, counterfactuals are emotional amplifiers that may result in social emotions of regret, guilt, or blame, as well as in the more positive emotions of relief or satisfaction (Byrne, 2002; Guttentag \& Ferrell, 2004; Johnson-Laird \& Byrne, 2002; Kahneman \& Miller, 1986).

In contrast to the amount of attention paid to counterfactual thinking, the study of counterfactual comprehension has been neglected by psycholinguists and discourse researchers. This neglect is not justifiable, because counterfactuals are produced not only to accomplish speakers' personal goals but also to be understood by addressees. The aim of this article is to explore the comprehension of counterfactuals embedded in texts using reading times and accessibility measures. In spite of the lack of specific research on the comprehension of counterfactuals, we will review literature relevant to the present subject. First, we will describe theoretical approaches to counterfactual meaning according to cognitive linguistics and the conditional reasoning literature; second, we will focus on the notion of updating, which is central to explaining the hypotheses of the present research; and finally we will describe studies on the comprehension of mentalist expressions that share characteristics with counterfactuals.

A popular hypothesis in cognitive linguistics is that understanding counterfactuals involves the representation of two possible states of the world (Fauconnier, 1994; Langacker, 1991). For instance, Fauconnier (1994) proposed that understanding counterfactuals requires building up both a mental space for the contents embedded in the counterfactual and a base space corresponding to reality.

M. de Vega, mdevega@ull.es 
Thus, when people read "If Mary had won the lottery, she would have bought a Mercedes car," they would keep in mind a mental space in which "Mary won the lottery and bought a Mercedes car" (p \& q) and a base-space interpretation in which "Mary did not win the lottery and did not buy a Mercedes car" (not-p \& not-q). Some entities in the counterfactual space correspond to entities in the base space. For instance, in the previous example the factual base space built by the addressee might include knowledge about Mary's actions, intentions, and wishes: She bought a lottery ticket last year; she dreams about being rich; she would like to be the owner of a Mercedes car; and so on. In turn, the mental space associated with the counterfactual meaning also includes some of these entities (Mary; the lottery; the Mercedes car), but arranged in a different way from in the "real" base space: Mary won the lottery and she was able to fulfill her dream of buying a Mercedes car. Fauconnier also proposed that there are links or connections between the homologous entities in the base space and the counterfactual space.

The dual nature of counterfactual meaning has also been explored empirically in the field of conditional reasoning (Byrne, 2002, 2005; Johnson-Laird \& Byrne, 2002; Santamaría, Espino, \& Byrne, 2005; Thompson \& Byrne, 2002). In most cases, these studies use off-line methods in which participants first read a conditional narrative in indicative (factual) or subjunctive (counterfactual) mood, and are then asked to choose from among several sentences those which are more consistent with, or are implied by, the conditional sentence's meaning. The results usually confirm that participants tend to choose exclusively a factual interpretation ( $\mathrm{p} \& \mathrm{q}$ ) for indicative conditionals, but that they choose both factual (p \& q) and counterfactual (not-p \& not-q) interpretations for subjunctive conditionals (see Thompson \& Byrne, 2002). Moving beyond this choice-task methodology, Santamaría et al. (2005) used a sentence priming paradigm, giving participants counterfactual conditionals such as "If there had been roses then there would have been lilies" as primes to understand target sentences like "There were roses and there were lilies," or "There were no roses and there were no lilies." The same procedure was used with factual conditional primes such as "If there are roses, there are lilies." When target reading times were collected, factual sentences primed only the affirmative target "There were roses and there were lilies," whereas counterfactual sentences primed both the affirmative and the negative targets, suggesting that readers had generated a double representation.

The above studies suggest that the meaning of counterfactuals is dual. However, many questions remain unclear, and to answer them may require a methodology more sensitive to online processes. If readers keep in mind two contradictory interpretations of a situation, what is the temporal course of their activation? Are counterfactual events integrated into the previous (factual) discourse, or are these events suppressed from the situation model? To answer such questions, the notion of updating developed by comprehension researchers might be useful. Updating takes place when readers modify their representation according to the evolving situation described by the text (e.g., de Vega, 1995; Glenberg, Meyer, \& Lindem, 1987; O'Brien, Cook, \& Peracchi, 2004; Zwaan \& Madden, 2004). Consequently, the most recent situation state from the protagonist's point of view (the here-and-now) should be more accessible in memory than are outdated situation states (Zwaan \& Madden, 2004). In some cases, the situation model unfolds matching the lineal structure of the text, but sometimes there is a mismatch between the situation here-and-now and the superficial text. For instance, some temporal adverbials prompt the readers to change in their situation model the order of the events described by the text (e.g., de Vega, Rinck, Díaz, \& León, 2007; Münte, Schiltz, \& Kutas, 1998), or to set a temporal discontinuity among events described consecutively in the text (e.g., Carreiras, Carriedo, Alonso, \& Fernández, 1997; Zwaan, 1996; Zwaan, Madden, \& Whitten, 2000). In these cases, the accessibility of the events is governed to some extent by their temporal status in the situation model. Thus, Zwaan et al. (2000) gave readers paragraphs such as "Mike was presenting his report. When he noticed Marsha enter the room, he glanced up and stopped/continued," and readers found that the probe PRESENTING was recognized faster in the "continued" than in the "stopped" version of the stories; that is, in the "continued" version, the event "presenting the report" was still in the situation here-and-now, whereas in the "stopped" version, readers" attention moved to a different situation state. This article explores whether counterfactuals embedded in narratives influence situation model updating. A simple hypothesis is that counterfactual events (which are not "real") are not incorporated into the ongoing situation model, and that the here-and-now therefore remains in events described previously in the text. However, updating processes could be more complex in counterfactuals than this hypothesis suggests, as we will see.

Mentalist expressions share with counterfactuals some characteristics of their meaning. First, like counterfactuals, mentalist sentences do not describe factual events, but refer to the character's mental attitudes (wishes or beliefs) toward a possible or hypothetical event. Consider, for instance: "John wishes that the yellow book were on the shelf." This sentence does not describe a factual situation but a protagonist's mental state. A proof of the special status of mentalist sentences is that their truth value cannot be easily established just by checking the perceptual environment (e.g., by looking for the yellow book on the shelf). Whether or not the yellow book is there is quite irrelevant for the reality of John's wishes. Second, comprehending mentalist sentences poses the computational problem of dealing with alternative situation models at the same time, which is also coincidental with the hypothetical dual meaning of counterfactuals. For instance, consider this sentence: "John wishes that the yellow book were on the shelf, but I know that it is in the suitcase." To understand such sentences, readers must be able to decouple two alternative situation models involving a particular event corresponding to John's belief and the speaker's belief (see, e.g., Leslie, 1987). Finally, when mentalist sentences are embedded in a factual text, the updating processes must be momentarily cancelled, because wishes 
and beliefs do not alter significantly the factual ongoing model (de Vega, Díaz, \& León, 1997; de Vega, Robertson, Glenberg, Kaschak, \& Rinck, 2004).

The last point is particularly relevant for the present research, because we hypothesize a similar disruption of updating when counterfactuals are embedded in narratives. Some studies with mentalist sentences embedded in narratives support the update-canceling hypothesis; that is, when a mentalist expression is read, the previous situation model is kept accessible and the updating process is temporarily cancelled. Thus, de Vega et al. (1997) demonstrated that readers of narratives are able to keep in mind the protagonist's false beliefs and to derive emotional inferences from them, in spite of the fact that the text provides new events (ignored by the protagonist) that change the emotional tone of the story. For instance, a story initially described Mary as waiting patiently $1 \mathrm{~h}$ for her boyfriend Charles in a café while she thought sympathetically about him. Later on, she called Charles's home and his mother informed her that he was playing poker with his friends. In another version of the story, Charles's mother did not inform Mary about this critical event, but the reader knows about it. Readers in the informed-protagonist version inferred that Mary was angry at the end of the story, whereas readers in the ignorant-protagonist version still inferred that Mary's mood was sympathetic. In other words, in the informed-protagonist story, readers update the protagonist's emotions, whereas in the ignorant-protagonist version they do not.

Similarly, readers in their minds clearly dissociate factual actions from planned actions. Thus, in one experiment, de Vega et al. (2004) presented readers with sentences describing a protagonist as performing two actions requiring similar body motions, such as, for instance, "painting a fence" and "chopping wood." The actions were described as being performed simultaneously or successively, by means of the adverbs while and after, respectively. The action in the second clause was preceded by a verbal periphrasis that was either factual (started to) or mentalist (thought of), as in the following examples:

(1) While painting a fence, the farmer started to chop the wood.

(2) After painting a fence, the farmer started to chop the wood.

(3) While painting a fence, the farmer thought of chopping the wood.

(4) After painting a fence, the farmer thought of chopping the wood.

The reading time for the second clause was longer for while factual sentences (sentence 1) than for after factual sentences (sentence 2), suggesting that readers have difficulty in understanding two incompatible actions described as simultaneous. However, sentences 3 and 4, with a mentalist periphrasis, were read equally fast for the while and the after conditions. One explanation is that the mental periphrasis, like counterfactual conditionals, is a mental space builder (Fauconnier, 1994) that prompts the reader to build a separate mental space decoupled from the factual situation model. Thus, the factual content and the mental content are not merged into a single simulation, but are kept as separate situation models.

Counterfactual sentences describe what might have been, not what is going on. In other words, the information provided by counterfactuals does not change the protagonist's here-and-now, and should not contribute to situation updating. However, given the dual meaning of counterfactuals, readers could momentarily consider counterfactual information as if it were "real." To integrate these apparently contradictory premises, we propose a modified update-canceling hypothesis. We predict that, immediately after reading a counterfactual sentence, readers will keep accessible both a factual ( $p \& q)$ and a counterfactual (not-p \& not-q) interpretation of the events. At this point, updating is not cancelled, because the "as if" interpretation and the negated interpretation of counterfactual events coexist. The two meanings, however, could not be kept in memory for long, and readers will suppress the "p \& q" meaning quite soon, maybe at the end of sentence wrap-up processes. When this happens, the reader's attention focuses on the preceding (nonupdated) situation model state, which remains quite accessible, whereas the events under the counterfactual scope become less accessible. This complex pattern contrasts with the comprehension of factual texts, in which a new piece of factual information determines the situation updating while the previous outdated information becomes less available.

\section{EXPERIMENT 1}

This experiment explores how sensitive readers of narratives are to the status of counterfactual information. Using a self-paced methodology, participants read short narratives with an embedded sentence describing a factual or counterfactual new event. A final sentence followed, which described either the outcome of the new event or the continuation of the previous situation (see Appen$\operatorname{dix}$ A). By collecting the final sentence reading time, and other measures, we investigated the idea that readers of counterfactual stories keep their attentional focus on the old situation, disregarding updating. The factual version of the task serves as a baseline condition, in which we expect the ordinary updating effects: The reader's attention moves to the new events. The predictions are that final sentences related to the new situation will be read faster in the factual than in the counterfactual context. By contrast, final sentences related to the old situation will be read faster in the counterfactual than in the factual context.

\section{Method}

Participants. Sixty psychology students (42 women) at the University of La Laguna participated in the experiment voluntarily, and received academic credit for their participation. All were native Spanish speakers.

Materials and Design. Forty-eight experimental stories were written in four versions each, resulting from combining the 2 context sentences (factual vs. counterfactual) and 2 final situations (new vs. old). Both experimental manipulations were within-participants. The structure of each story displayed in Appendix A was as follows: (1) a paragraph introducing the protagonist and describing an initial event (listening to the radio to know the winning lottery number); (2) a context sentence describing a new event (winning the prize) in 
either a factual or a counterfactual format; (3) a subordinate clause common to the factual and counterfactual context (buying a Mercedes car); (4) a final sentence describing an event related either to the old situation (tearing the ticket to pieces and cleaning the kitchen) or to the new event (getting into the car and feeling like a queen); (5) a coherence judgment prompt; and (6) a test probe word. The stories were all in Spanish. The final sentence included two clauses. The first clause (hereinafter critical clause) always had a similar structure (protagonist name + verb + complement), was always six words long, and was followed by the conjunction "and" and a second clause of variable length (hereinafter, the postcritical clause). Notice that two versions of each story were consistent ("counterfactual \& old" and "factual \& new"), and two were inconsistent ("counterfactual \& new" and "factual \& old"). In addition, 48 filler stories ( 32 of them with the same structure as the experimental ones) and 4 practice stories were written.

Norming. The 48 experimental stories were selected from a larger sample of 80 stories in a norming study. The 80 stories were written in all four experimental versions, and 16 booklets of 20 stories were built with these, including in each booklet 5 factual-old, 5 factual-new, 5 counterfactual-old, and 5 counterfactual-new stories. One hundred sixty students (none of whom participated in the main experiment) received the booklets (10 participants received each booklet) and were asked to judge on a 7-point scale how sensible each story was. Stories rated with 5 or more in the consistent versions and 3 or less in the inconsistent versions were selected for the main study ( 36 stories met this criterion in all four versions). The remaining 12 stories selected for the experiment met the criterion in three of their versions, and the "wrong" versions were corrected by the experimenters and a group of judges.

Participants rated as more sensible those stories categorized as consistent by the experimenters (factual-new: $M=5.49, S D=.83$; and counterfactual-old: $M=4.82, S D=1.01)$ than the stories categorized as inconsistent (factual-old: $M=2.93, S D=1.00$; counterfactualnew: $M=2.75, S D=.82$ ). These differences were statistically significant as shown by the context $\times$ final situation interaction $\left[F_{2}(1,79)=\right.$ 299.99, $\left.M S_{\mathrm{e}}=1.43, p<.0001\right]$. The sensibility rates did not correlate significantly with the reading times or the judgment times from the experiment; hence, that variable was not considered an effective covariate. However, sensibility rates negatively correlated with judgment errors in the factual-old $(r=-.43, p<.001)$ and counterfactual-new $(r=-.36, p<.03)$ conditions. These correlations could derive from the fact that both the sensibility rates and the judgment errors are indices of metacognitive processes in comprehension.

Procedure. Each participant was assigned one of four lists of materials, resulting from counterbalancing the different versions of the experimental stories. Thus, each participant received as experimental stories 12 counterfactual-old, 12 counterfactual-new, 12 factualold, and 12 factual-new. The practice and the filler sentences were the same for all participants. At the beginning of the session, participants received the experimental instructions and the 4 practice stories, followed by the whole set of experimental and filler stories in random order. They were instructed to use a self-paced methodology to read each story sentence by sentence at normal speed for compre- hension. At the end of each story they received the prompt "WAS IT COHERENT?" and they had to make a speeded YES/NO judgment by pressing the P or Q key in the keyboard, respectively. Immediately afterwards they were given a test probe consisting of a word they had to identify as belonging or not to the story. They again used the assigned YES/NO keys in the keyboard.

The reading times for the common, the critical, and the postcritical clauses, and the coherence judgment times, the test probe times were collected, along with the accuracy of judgments and test probe responses.

\section{Results}

Six participants were dismissed from the analyses because they had an error rate higher than 30\% in their coherence judgments. Participant data corresponding to judgment errors ( $16 \%$ of data), as well as clause reading times longer than 3,500 msec or shorter than $300 \mathrm{msec}$, were also dismissed from the analyses (about $2 \%$ of data). The results were submitted to $2 \times 2$ ANOVAs with context (factual/ counterfactual) and final situation (new/old) as withinparticipants factors in the by-participants analysis $\left(F_{1}\right)$, and as within-stories factors in the by-stories analysis $\left(F_{2}\right)$.

Critical clause reading time. Mean reading times are shown in Table 1 . The only significant effect was the context $\times$ final situation interaction $\left[F_{1}(1,53)=8.60, M S_{\mathrm{e}}=\right.$ $27,133, p<.005 ; F_{2}(1,47)=5.66, M S_{\mathrm{e}}=71,522, p<$ $.02]$. This interaction resulted from the advantage of consistent over inconsistent conditions. Specifically, participants read the old situation clause faster in the counterfactual than in the factual context $\left[t_{1}(53)=3.07, p<.003\right.$; $\left.t_{2}(47)=2.46, p<.02\right]$; however, the expected advantage of the new situation clause in the factual context was not obtained $\left[t_{1}(53)=1.00\right.$, n.s.; $t_{2}(47)=1.13$, n.s. $]$. A possible explanation is that the factual context was interpreted exclusively as factual and the old situation clause was immediately detected as conflicting, increasing the reading time. In contrast, the counterfactual context might be temporarily interpreted both as counterfactual and as factual, and thus the old and the new situation clauses were read rapidly. However, the two interpretations of counterfactuals were unbalanced, because old situation clauses were read faster than were new situation clauses in counterfactual stories $\left[t_{1}(53)=2.5, p<.01 ; t_{2}(47)=1.9, p=\right.$ $.06]$, suggesting that the counterfactual interpretation was already dominant at this point.

Postcritical clause reading time. Mean reading times are shown in Table 1. Again there was a context $\times$ final situation interaction $\left[F_{1}(1,53)=36.13, M S_{\mathrm{e}}=27,695\right.$,

Table 1

Experiment 1: Critical Clause and Postcritical Clause Mean Reading Times

(in Milliseconds) and Standard Deviations As a Function of Context (Factual vs. Counterfactual) and Final Situation (New vs. Old)

\begin{tabular}{|c|c|c|c|c|c|c|c|c|}
\hline & \multicolumn{4}{|c|}{ Critical Clause } & \multicolumn{4}{|c|}{ Postcritical Clause } \\
\hline & \multicolumn{2}{|c|}{ New Situation } & \multicolumn{2}{|c|}{ Old Situation } & \multicolumn{2}{|c|}{ New Situation } & \multicolumn{2}{|c|}{ Old Situation } \\
\hline & $M$ & $S D$ & $M$ & $S D$ & $M$ & $S D$ & $M$ & $S D$ \\
\hline Factual (F) & 2,129 & 365 & 2,198 & 400 & 1,523 & 307 & 1,611 & 337 \\
\hline Counterfactual (C) & 2,190 & 372 & 2,093 & 365 & 1,668 & 369 & 1,483 & 368 \\
\hline $\mathrm{F}-\mathrm{C}$ difference & -61 & & $105^{* *}$ & & $-145^{*}$ & & $128^{* *}$ & \\
\hline
\end{tabular}


$\left.p<.0001 ; F_{2}(1,47)=16.45, M S_{\mathrm{e}}=53,499, p<.0001\right]$. The interaction consisted again in the advantage of the consistent over the inconsistent conditions. In this case, however, the two components of the interaction were significant; that is, old situation clauses were read faster in the counterfactual context $\left[t_{1}(53)=2.87, p<.006 ; t_{2}(47)=\right.$ $3.39, p<.001]$, and new situation clauses were read faster in the factual context $\left[t_{1}(53)=4.95, p<.0001 ; t_{2}(47)=\right.$ $1.82, p=.07]$. Given that the length of the postcritical clause was variable across items, we used the number of syllables as covariant in a new item analysis, and the interaction of context $\times$ final situation was still highly significant $\left[F_{2}(1,47)=13.41, M S_{\mathrm{e}}=51,051, p<.001\right]$. In other words, there was a spillover effect on the postcritical sentence reading time, even after discounting the effect of sentence length.

Coherence judgment time. The mean response times (RTs) are shown in Table 2. The effect of final situation was significant $\left[F_{1}(1,53)=4.98, M S_{\mathrm{e}}=31,223, p<.03\right.$; $\left.F_{2}(1,47)=5.78, M S_{\mathrm{e}}=32,793, p<.02\right]$. Participants were faster in their judgments when the final clause was in the new situation $(1,161 \mathrm{msec})$ than when it was in the old situation version $(1,215 \mathrm{msec})$. However, this effect was qualified by interaction with context $\left[F_{1}(1,53)=6.12\right.$, $M S_{\mathrm{e}}=67,867, p<.02 ; F_{2}(1,47)=4.32, M S_{\mathrm{e}}=25,698$, $p<.04]$. The most salient aspect of this interaction was that responses to the (consistent) counterfactual-old situation condition were $142 \mathrm{msec}$ slower than were responses to the (inconsistent) counterfactual-new situation condition $\left[t_{1}(53)=2.89, p<.005 ; t_{2}(47)=2.73, p<.009\right]$.

Judgment errors. Participants made more errors in stories with an old situation than in those with a new final situation $\left[F_{1}(1,53)=7.17, M S_{\mathrm{e}}=108, p<.01 ; F_{2}(1,47)=3.92\right.$, $\left.M S_{\mathrm{e}}=255, p=.053\right]$, although the effect was qualified by the interaction with context $\left[F_{1}(1,53)=5.39, M S_{\mathrm{e}}=318\right.$, $\left.p<.02 ; F_{2}(1,47)=3.61, M S_{\mathrm{e}}=230, p=.06\right]$. Participants made more errors in the consistent than the inconsistent conditions; the number of errors was, therefore, higher in the counterfactual-old situation than in the counterfactual-new situation stories $\left[t_{1}(53)=3.26, p<.002 ; t_{2}(47)=2.85, p<\right.$ $.006]$, and in the factual-new situation than in the factualold situation condition $\left[t_{1}(53)=3.08, p<.003 ; t_{2}(47)=\right.$ 0.77, n.s.]. There was no speed-accuracy trade-off: Those conditions that produced slower judgments were also associated with more errors (Table 2).

When common clause reading times, probe RTs, or probe errors were used as dependent measures, no statistically significant effects were obtained.

\section{Discussion}

In this experiment, readers were given a factual or counterfactual context, followed immediately by a final sentence that was an appropriate continuation to one of the contexts. The data showed a clear consistency effect in both the factual and the counterfactual version of the stories. Namely, when the final sentence was a reinstatement of the initial situation (previous to the context sentence), reading was considerably faster in the counterfactual than in the factual version. However, when the final sentence was a completion of an ongoing situation, reading was faster in factual than in counterfactual contexts. The latter claim has to be qualified, however. As mentioned above, the reading times of the critical clause suggest that immediately after a reader finished reading the counterfactual sentence, both a factual and a counterfactual interpretation of the events were momentarily accessible. Only during the postcritical clause does the factual interpretation of counterfactuals fade. In sum, readers of factual texts update the situation model as expected, whereas readers of counterfactual texts seem to perform a more sophisticated process. Initially, while reading the critical clause, they could maintain a double representation: an updated situation model (as if the counterfactual event was factual) and a nonupdated situation model (moving back to the initial situation). Later on, while the postcritical clause is being read, only the nonupdated situation model remains.

Whereas the reading time data provide a clue to comprehension processes, the semantic judgment data provide complementary information about readers' metacognitive processes. The most remarkable effect was the relatively poor performance in the consistent conditions. Particularly, readers' performance was worse (slower and less accurate responses) for counterfactual-old situation stories (consistent) than for counterfactual-new situation stories (inconsistent); and readers were also less accurate in their coherence judgments for factual-new (consistent) than for factual-old situation stories. A tentative explanation of these facts is that inconsistent stories are easier to judge, because they involve a clear contradiction. Consider, for instance, the lottery story. In the counterfactual inconsistent version, after reading "If Mary had won the lottery prize, she would have bought a Mercedes car," participants would be surprised to know that "Mary got into the Mercedes car and felt like a queen," and when they receive the judgment prompt they would be ready to choose immediately the "no" response. In the same vein, the factually inconsistent story, in which after winning the lottery, "Mary

Table 2

Experiment 1: Mean Response Times (in Milliseconds) With Standard Deviations for Coherence Judgments, and Percentages of Error (\%) As a Function of Context (Factual vs. Counterfactual) and Final Situation (New vs. Old)

\begin{tabular}{|c|c|c|c|c|c|c|c|c|c|c|}
\hline & \multicolumn{4}{|c|}{ New Situation } & \multicolumn{4}{|c|}{ Old Situation } & \multicolumn{2}{|c|}{$\begin{array}{l}\text { New - Old } \\
\text { Difference }\end{array}$} \\
\hline & $M$ & $S D$ & $\%$ & $S D$ & $M$ & $S D$ & $\%$ & $S D$ & $M$ & $\%$ \\
\hline Factual & 1,195 & 357 & 18 & 14 & 1,161 & 301 & 16 & 11 & 34 & $4^{*}$ \\
\hline Counterfactual & 1,127 & 307 & 10 & 10 & 1,269 & 370 & 19 & 16 & $-142^{* *}$ & $-9^{* *}$ \\
\hline
\end{tabular}

*Significant by participants $(p<.01) ;{ }^{* *}$ significant by participants $(p<.01)$ and by stories $(p<.01)$. 
tore the ticket to pieces and started to clean the kitchen," can be judged immediately as nonsense. By contrast, consistent stories could be relatively difficult to judge, because there are potentially many consistent endings to a story. This is particularly true for counterfactual sentences, which are usually associated with intense protagonist emotions such as frustration, guilt, regret, and so on (Byrne, 2002; Guttentag \& Ferrell, 2004; Johnson-Laird \& Byrne, 2002; Kahneman \& Miller, 1986). However, none of the current counterfactual consistent stories ended with a description of such likely emotional consequences, but rather described emotionally neutral events. Our participants might have felt that our consistent endings were, at least, incomplete, and thus delayed their judgments, or even considered some of them inconsistent.

\section{EXPERIMENT 2}

Experiment 1 demonstrated that people performing a reading task were sensitive to the special status of counterfactual information, and also that they were aware of the consistency or inconsistency of information following the counterfactual context. The present experiment moves one step further, to explore how readers' attentional focus shifts when they read factual and counterfactual information embedded in stories. The hypotheses are as follows:

For factual stories, the readers' attentional focus moves from the initial factual situation to the new factual situation as a consequence of updating. For instance, in the factual version of the story in Appendix B, readers initially focus on the office scenario and the protagonist's typing activity. When readers process the new factual information, their attention moves to the café scenario and the protagonist's drinking action. A consequence of the updating process is that at the end of the story, the initial factual information should become less accessible and the new factual information should be highly accessible (this updating effect should not be confused with a simple recency effect, as we will see).

By contrast, for counterfactual stories, when readers start to read they focus on the initial factual scenario (e.g., the office). When they find the counterfactual scenario of the café, the events in this scenario do not influence the updating of the initial scenario (office), because counterfactual and factual information are not mixed. Consequently, the initial factual representation remains in focus and will be relatively accessible at the end of the story. But what is the fate of counterfactual information? It could be immediately suppressed and become less accessible because it does not contribute to situation updating. Another possibility we mentioned before is that counterfactual events could be momentarily represented as factual and could continue to be accessible at the end of the story. To test this updatecanceling hypothesis, we used a probe identification task presented immediately after the critical (factual or counterfactual) sentence. The purpose of the probe was to measure the accessibility of a verb corresponding to the initial scenario (e.g., type), or to a clause subordinated to the critical sentence (e.g., drink). Note that this clause (e.g., "to drink a beer") was shared by both versions of the story in order to minimize the influence of the local grammatical structure.

\section{Method}

Participants. Forty psychology students ( 33 women) at the University of La Laguna participated in the experiment voluntarily and received academic credit for their participation. All were native Spanish speakers, and none had participated in the previous experiment.

Materials and Design. Forty stories from the previous experiment were modified to fit the purpose of the present experiment (see Appendix B). There were four versions of each story resulting from combining 2 contexts (factual/counterfactual) and 2 targets (initial/final). The two factors were manipulated within participants. In addition, 40 filler stories with a similar structure and factual/ counterfactual manipulation were employed, all of which included false test probes. Finally, there were 4 practice stories.

Procedure. Participants were assigned one of four lists of materials resulting from counterbalancing context and target. They received instructions to read each story for comprehension, and to decide as fast and accurately as possible whether a test probe word was present in the story. At the end of the story they received a yes/ no memory question. The yes response was assigned to the "p" key (marked YES) and the no response to the "q" (marked NO) on the keyboard. After the instructions, they were given the practice stories and the whole set of experimental and filler stories, which were presented randomly. Each trial started with the prompt "NEW TEXT," which remained on the screen for $500 \mathrm{msec}$. A 100 -msec blank, a 500-msec fixation point in the middle of the screen, and a 500msec blank followed. Then the main part of the story was presented, clause by clause, in the middle of the screen, at a rate of $1,500 \mathrm{msec}$ per clause, with each clause followed by a 100 -msec blank. Then the test probe appeared in upper case and enhanced by asterisks at both sides of the word. The probe remained on the screen for up to $5,000 \mathrm{msec}$, or until the participant's response. Two additional clauses completed the story; finally, there was a Yes/No question that referred $1 / 3$ to the initial part of the story, $1 / 3$ to the critical content, and $1 / 3$ to the end of the story. Half of the test probes and half of the questions were false.

\section{Results}

Four participants were dismissed from the analyses because they had an error rate higher than $33 \%$ in response to test probes. Participants' data corresponding to probe errors ( $15 \%$ of the data) as well as RTs longer than $3,500 \mathrm{msec}$ or shorter than $300 \mathrm{msec}$ were also discarded from the RT analyses (about $1 \%$ of the data). In addition, data from three stories were excluded because the experimenters had mistakenly changed the verb tense of the probe (e.g., to "eating" from the target "eat"); consequently, more than $40 \%$ of participants failed to recognize it. In this experiment and in the next one, the results were submitted to $2 \times 2$ ANOVAs with context (factual/counterfactual) and target (initial/final) as within-participants factors in the by-participants analysis $\left(F_{1}\right)$, and as within-stories factors in the by-stories analysis $\left(F_{2}\right)$.

Probe RTs. The mean RTs are shown in Table 3. There was a strong effect of target $\left[F_{1}(1,35)=48.73, M S_{\mathrm{e}}=\right.$ $58,385, p<.0001 ; F_{2}(1,36)=78.80, M S_{\mathrm{e}}=101,752, p<$ $.0001]$, as the initial target was responded to much slower $(1,546 \mathrm{msec})$ than was the final target $(1,265 \mathrm{msec})$. However, this main effect of target was qualified by the important interaction with context $\left[F_{1}(1,35)=5.71, M S_{\mathrm{e}}=\right.$ $34,153, p<.02 ; F_{2}(1,36)=4.14, M S_{\mathrm{e}}=39,286, p<$ 
.05]. The main component of this interaction was that the initial target was recognized faster in the counterfactual than in the factual stories $\left[t_{1}(35)=2.12, p<.03 ; t_{2}(36)=\right.$ 2.76, $p<.009]$, although no significant difference between factual and counterfactual stories for the final target was obtained $\left[t_{1}(33)=1.02\right.$, n.s.; $t_{2}(36)=0.16$, n.s. $]$.

Probe errors. The only significant effect was the target $\left[F_{1}(1,35)=53.05, M S_{\mathrm{e}}=191, p<.0001 ; F_{2}(1,36)=\right.$ 65.52, $\left.M S_{\mathrm{e}}=163, p<.0001\right]$. As for RTs, participants made many more errors for initial than for final targets.

As to the performance on the questions, there was no significant effect either in RT (grand mean: $1,998 \mathrm{msec}$ ) or in errors (grand mean: 12\%).

\section{Discussion}

The experiment partly confirmed the update-canceling hypothesis: The initial situation was more accessible at the end of the story in the counterfactual than in the factual version, because in the former the reader's attention moves back to the previous scenario. As for the most recent information, it was equally accessible in factual and in counterfactual stories. A possible explanation is that the recency of the new situation target in the previous discourse might prevent any advantage from accruing from the factual stories, because in both factual and counterfactual stories, recent information would still be active in working memory at the probe time.

This explanation is not very plausible, however, as accessibility effects for probes referring to recently-negated concepts have been reported in the literature (e.g., Kaup, 2001; Kaup \& Zwaan, 2003; MacDonald \& Just, 1989). For instance, immediately after reading the sentence "Mary bakes bread but not cookies," the probe cookies was less accessible than the probe bread (MacDonald \& Just, 1989). Generally speaking, the negation operator suppresses the information within its scope very early, in the range of milliseconds (e.g., Kaup \& Zwaan, 2003). By analogy, we may argue that a counterfactual expression is an implicit negation of an antecedent and a consequent (not-p \& not-q) and that, consequently, a similar early suppression could be expected for the contents within its scope. Therefore, the high accessibility of counterfactual contents might obey other causes beyond recency.

A more substantial interpretation of the accessibility of recent information in counterfactual stories is that after reading the counterfactual sentence, participants momentarily activated the current events ( $\mathrm{p} \& \mathrm{q})$, producing a partial updating before suppressing them (not-p \& not-q) at a later moment. Some results of Experiment 1 are also consistent with this explanation: Updated continuation clauses ( $p \&$ q) were read equally fast, whether they were in counterfactual or factual contexts. As we have seen, these results are compatible with the dual nature of counterfactual meaning described in both the literature on conditional reasoning and that on mental spaces.

In sum, our results suggest that immediately after reading a counterfactual sentence embedded in a short story, participants keep the initial information foregrounded. However, at this early stage readers do not suppress the counterfactual events, and they have access to them as if the events were factual. The reader's state of mind seems quite paradoxical, as the factual information and the counterfactual information coexist and are highly accessible but are not mixed to the point of producing complete situation model updating.

\section{EXPERIMENT 3}

Experiment 2 showed that after a counterfactual sentence is read, the preceding information is more accessible than it is after a factual sentence is read, whereas the final information was equally accessible in factual and in counterfactual formats. These phenomena are consistent with the update-canceling hypothesis of counterfactual comprehension. However, we also postulated that, in a second stage, information embedded in a counterfactual sentence would be less accessible than the same information within a factual sentence would be. To observe this suppression of counterfactual events, it might be necessary to measure target accessibility at a later point. In the present experiment, the probe test was considerably distanced from the target information in the critical sentence. First, the order of the clauses in the critical sentence was changed so that the subordinate clause with the target verb (e.g., "to drink a beer") was not in the last-sentence position. Second, a neutral filler sentence was placed immediately before the probe (e.g., "The day was quite hot"). Appendix C shows an example of materials rewritten for this experiment.

\section{Method}

Participants. Seventy-seven psychology students ( 59 women) at the University of La Laguna participated in the experiment voluntarily, and received academic credit for their participation. All were native Spanish speakers, and none had participated in the previous experiments.

Materials and Design. The experimental design was identical to the previous one, namely, a 2 context $\times 2$ target position within-

Table 3

Experiments 2 and 3: Mean Response Times (in Milliseconds) With Standard Deviations and Percentages of Error (\%) for Test Probes As a Function of Context and Target

\begin{tabular}{|c|c|c|c|c|c|c|c|c|c|c|c|c|c|c|c|c|}
\hline & \multicolumn{8}{|c|}{ Experiment 2} & \multicolumn{8}{|c|}{ Experiment 3} \\
\hline & \multicolumn{4}{|c|}{ Initial Target } & \multicolumn{4}{|c|}{ Final Target } & \multicolumn{4}{|c|}{ Initial Target } & \multicolumn{4}{|c|}{ Final Target } \\
\hline & $M$ & $S D$ & $\%$ & $S D$ & $M$ & $S D$ & $\%$ & $S D$ & $M$ & $S D$ & $\%$ & $S D$ & $M$ & $S D$ & $\%$ & $S D$ \\
\hline Factual (F) & 1,598 & 487 & 24 & 17 & 1,243 & 367 & 7 & 8 & 1,475 & 363 & 20 & 15 & 1,347 & 279 & 14 & 12 \\
\hline Counterfactual (C) & 1,494 & 394 & 24 & 16 & 1,286 & 303 & 7 & 9 & 1,477 & 323 & 18 & 14 & 1,468 & 389 & 12 & 12 \\
\hline $\mathrm{F}-\mathrm{C}$ difference & $104^{* *}$ & & 0 & & -43 & & 0 & & -2 & & 2 & & $-121^{* *}$ & & 2 & \\
\hline
\end{tabular}

${ }^{* *}$ Significant by participants $(p<.03)$ and by stories $(p<.01)$. 
participants design. The materials were the same as in Experiment 2, except that the clause order was changed in the critical sentence and the probe test was placed after a filler sentence.

Procedure. The procedure was the same as in the previous experiment.

\section{Results and Discussion}

Applying the same criterion as in the previous experiment, 16 out of 77 participants were dismissed because they had an error rate higher than $33 \%$ in the probes. The poor performance of $1 / 5$ of the participants was probably due to the greater memory demands of the task. In the new texts, the probe was quite distant from both the initial and the final target as a consequence of the new structure of the materials. The same data-trimming procedures and statistics as those in Experiment 2 were used with the remaining 61 participants.

Probe RTs. The mean RTs are shown in Table 3. Again, there was a strong effect of target position $\left[F_{1}(1,60)=9.86\right.$, $M S_{\mathrm{e}}=29,201, p<.003 ; F_{2}(1,39)=11.6, M S_{\mathrm{e}}=45,687$, $p<.002]$, as the initial probe was responded to more slowly $(1,476 \mathrm{msec})$ than the final probe $(1,407 \mathrm{msec})$. However, the effect of target position was qualified by the interaction with the context $\left[F_{1}(1,60)=7.00, M S_{\mathrm{e}}=\right.$ $\left.30,966, p<.01 ; F_{2}(1,39)=4.4, M S_{\mathrm{e}}=17,330, p<.04\right]$. The main component of this interaction was that the final target was recognized faster in the factual than in the counterfactual story $\left[t_{1}(60)=3.25, p<.002 ; t_{2}(39)=3.6\right.$, $p<.001]$. The withdrawal of the probe from the second position target resulted in the emergence of the advantage of factual over counterfactual information. Thus, another prediction of the update-canceling hypothesis was supported: Information included in a counterfactual sentence becomes less accessible than does the same information embedded in a factual sentence; this suggests a later suppression process. As was not the case in Experiment 2, the initial target was equally accessible at the end of both the counterfactual and the factual stories $\left[t_{1}(60)<1\right]$. This could be easily explained, because - even in the counterfactual stories - the reader's attention may start to move to the recent filler information.

Probe errors. The only significant effect was the target $\left[F_{1}(1,60)=12.36, M S_{\mathrm{e}}=162, p<.001 ; F_{2}(1,39)=\right.$ $\left.15.5, M S_{\mathrm{e}}=164, p<.0001\right]$. Consistent with the RT performance, participants made more errors for initial (19\%) than final $(13 \%)$ targets.

As to performance on the questions, there was no significant effect on either RT (grand mean: 2,167 msec) or errors (grand mean: 14\%).

\section{GENERAL DISCUSSION}

The present set of experiments aimed to explore how updating processes are modified when counterfactual contents are embedded in narratives. Several predictions were upheld. First, the reading time data of Experiment 1 clearly showed that readers are immediately sensitive to the special status of counterfactuals. In comparison with factual stories, readers of counterfactual stories read faster nonupdated continuations (referring to the old situation) and slower updated continuations (referring to the new situation). Second, information belonging to the initial part of the story became less accessible after a factual event was read, but remained highly accessible after a counterfactual event was read (Experiment 2). By contrast, in some circumstances, events described in a factual sentence were more accessible than were the same events described in a counterfactual counterpart (Experiment 3). Third, the above statements have to be qualified by the fact that after reading a counterfactual, a factual interpretation of the events is activated and momentarily coexists with the nonfactual interpretation.

The latter claim is consistent with cognitive linguistic theories (Fauconnier, 1994; Langacker, 1991) and conditional reasoning data (e.g., Byrne, 2002, 2005; JohnsonLaird \& Byrne, 2002; Santamaría et al., 2005; Thompson \& Byrne, 2002), which assume that readers of counterfactuals build a double representation, the "p \& q" meaning and the "not-p \& not-q" meaning. The first meaning is an "as if" simulation of the scenario embedded in the counterfactual. The negative "not-p \& not-q" meaning involves readers' moving their attention away from the current information. The present study supports this proposal but, in addition, provides a more articulated view of the temporal course of dual meaning. The two meanings seem to coexist in working memory for a while, as the reading time (Experiment 1) and the accessibility data (Experiment 2) suggest. First, the high accessibility of the initial scenario preceding the counterfactual suggests that readers have processed the implicit negation "not-p \& not-q" and have reinstated the initial situation model backwards. Second, the relatively high accessibility of the final scenario indicates that the simulation of the current " $p \& q$ " situation had also been constructed. However, the latter simulation is a short-lived phenomenon, as Experiment 3 demonstrated. When additional clauses are read after the counterfactual information, the " $p$ \& q" events become less accessible, suggesting an inhibitory process, as we will see later. This pattern contrasts with that of factual stories, in which ordinary updating takes place and attention moves "forward" from the initial situation to the final situation.

This article does not provide direct cues about the mechanisms of dual meaning in counterfactuals. Why does the "as if" simulation occur in the first place? One reason is pragmatic: Counterfactual information consists precisely of "undoing reality" and imagining an alternative (e.g., Roese, 1997, 2005). The contrast between what happened and what might have happened is the basis for the main functional characteristics of counterfactuals such as causal reasoning, the activation of emotions, and so on. Although this explanation is reasonable, it seems rather circular and does not account for the time course of both the "as if" simulation and the negative meaning with a reinstatement of the initial scenario. Let us consider, then, another explanation: Counterfactuals are implicit double negations, and implicit negations, which are difficult to process, demand extra time (Clark \& Chase, 1972; Kaup \& Zwaan, 2003). As a consequence, readers have time to process the counterfactual events as a faster "as if" simu- 
lation, which could be considered an opportunistic byproduct of the slow processing of implicit negations.

The present research confirms the dual meaning of counterfactuals suggested by the reasoning literature (Byrne, 2002, 2005; Johnson-Laird \& Byrne, 2002; Santamaría et al., 2005; Thompson \& Byrne, 2002). However, it also shows that the counterfactual events are suppressed after a while, in spite of the fact that they had been activated in working memory recently (Experiments 2 and 3 ). The suppression of competing information is a process usually attributed to the prefrontal cortex, so we might expect prefrontal patients to have difficulties in the processing of counterfactuals. The neuroscience literature confirms this idea. Patients with prefrontal cortex lesions show impairment in a counterfactual thought generation task (Gomez-Beldarrain, Garcia-Monco, Astigarraga, Gonzalez, \& Grafman, 2005); patients with orbitofrontal lesions do not experience regret, a typical "counterfactual" emotion, when they play decision games (Camille et al., 2004); and Parkinson's patients show functional deficits both in the frontal cortex and in counterfactual generation (McNamara, Durso, Brown, \& Lynch, 2003).

If suppression is a critical process underlying counterfactual processing, we could also expect a selective difficulty in the comprehension of counterfactuals associated with aging, because older adults show a decline in inhibitory processes that could affect their memory for texts. For instance, Radvansky, Zacks, and Hasher (2005) have shown how young and old adults differ in inhibition, using a fan effect paradigm. When a concept was described in several competing scenarios (e.g., "the potted palm is in the airport," "the potted palm is in the hotel," "the potted palm is in the movie theater"), the recognition of a probe sentence (e.g., "the potted palm is in the airport") was delayed because there was interference. To solve this interference, participants have to inhibit the competing scenarios (the hotel and the movie theater) and, consequently, when a new probe involving an inhibited scenario is given (e.g., "the oak is in the hotel"), a further response delay is observed, in this case due to inhibition rather than to interference. Both young and old adults showed interference in the first kind of probe, but only young adults showed inhibition in the second probe, because inhibitory processes are less conspicuous in the elderly. An interesting research avenue could be to run experiments like the present ones with older readers. A possible hypothesis is that old adults will keep counterfactual events highly accessible, even under the delayed probe conditions of Experiment 3, because of a failure to inhibit them.

Another issue that merits further research is whether counterfactual information activates embodied meaning or is an ungrounded representation (e.g., symbolic, amodal, abstract). The "as if" interpretation of a counterfactual could be associated with a perceptual simulation of its content, as suggested by a recent study (Urrutia, Moreno, de Vega, \& Morera, 2005). Readers were given either factual or counterfactual sentences with an embedded clause describing a transfer away from me (e.g., "Because I am generous I've lent the Harry Potter novel to my brother") or toward me (e.g., "If my brother had been generous, he would have lent me the Harry Potter novel"). The sentences were presented auditorily for comprehension, except for the transfer verb (i.e., lent), which was presented visually on the screen. The verb was animated to produce an apparent motion away or toward the participants, whose task was to move their index finger in the same direction as the animated word and to press a key. For factual sentences, the motor response was faster in the mismatching conditions (e.g., away transfer-away animation) than in the matching conditions (e.g., toward transfer-away animation), confirming other results in the literature (e.g., Kaschak et al., 2005). This interference in the matching conditions may be due to the fact that transfer sentences activate an embodied simulation that recruits the same brain areas involved in perceiving motion. What is more important, the same mismatching advantage was obtained for counterfactual sentences; this is consistent with an embodied simulation of the "as if" meaning of the sentence.

Given the psychological prominence of counterfactual thinking, it seems necessary for psycholinguists, and discourse and memory researchers, to try to fill the knowledge gap about how these linguistic structures are understood. This article reveals some remarkable phenomena on this subject: Counterfactuals cancel discourse updating in spite of the fact that a factual interpretation of their content can be momentarily accessible. Further studies might be necessary to explore in more detail the comprehension of counterfactuals, the temporal course of dual meaning activation, the embodied (or disembodied) nature of dual meanings, the role of inhibitory processes, the genesis of emotional states and inferences, and the brain bases of counterfactuals.

\section{AUTHOR NOTE}

This research was supported by the Spanish Ministry of Education and Science Grant BS02002-04301-C02-02 to M.d.V., and by Ministry of Education of Chile MECESUP Grant UCV0004, UCO to B.R. Correspondence concerning this article should be addressed to $M$. de Vega, Departamento de Psicología Cognitiva, Universidad de La Laguna, Campus de Guajara, 38205 La Laguna, Canary Islands, Spain (e-mail: mdevega@ull.es).

\section{REFERENCES}

Byrne, R. M. J. (2002). Mental models and counterfactual thoughts about what might have been. Trends in Cognitive Sciences, 6, 426-431.

BYRNE, R. M. J. (2005). The rational imagination: How people create alternatives to reality. Cambridge, MA: MIT Press.

Camille, N., Coricelli, G., Sallet, J., Pradat-Diehl, P., Duhamel, J.-R., \& SiRIGU, A. (2004). The involvement of the orbitofrontal cortex in the experience of regret. Science, 304, 1167-1170.

Carreiras, M., Carriedo, N., Alonso, M. A., \& Fernández, A. (1997). The role of verb tense and verb aspect in the foregrounding of information during reading. Memory \& Cognition, 25, 438-446.

Clark, H. H., \& Chase, W. G. (1972). On the process of comparing sentences against pictures. Cognitive Psychology, 3, 472-517.

DE VEGA, M. (1995). Backward updating of mental models during continuous reading of narratives. Journal of Experimental Psychology: Learning, Memory, \& Cognition, 21, 373-385.

DE Vega, M., Díaz, J. M., \& LEÓN, I. (1997). To know or not to know: Comprehending protagonists' beliefs and their emotional consequences. Discourse Processes, 23, 169-192. 
de Vega, M., Rinck, M., Díaz, J. M., \& León, I. (2007). Figure and ground in temporal sentences: The role of the adverbs when and while. Discourse Processes, 43, 1-23.

de Vega, M., Robertson, D. A., Glenberg, A. M., KaschaK, M. P., \& RINCK, M. (2004). On doing two things at once: Temporal constraints on actions in language comprehension. Memory \& Cognition, 32, 1033-1043.

FAUCONNIER, G. (1994). Mental spaces: Aspects of meaning construction in natural language. New York: Cambridge University Press.

Glenberg, A. M., Meyer, M., \& Lindem, K. (1987). Mental models contribute to foregrounding during text comprehension. Journal of Memory \& Language, 26, 69-83.

Gomez-Beldarrain, M., Garcia-Monco, J. C., Astigarraga, E., GonZalez, A., \& Grafman, J. (2005). Only spontaneous counterfactual thinking is impaired in patients with prefrontal cortex lesions. Cognitive Brain Research, 24, 723-726.

Guttentag, R., \& Ferrell, J. (2004). Reality compared with its alternatives: Age differences in judgments of regret and relief. Developmental Psychology, 40, 764-775.

Harris, P. L., German, T., \& Mills, P. (1996). Children's use of counterfactual thinking in causal reasoning. Cognition, 61, 233-259.

Johnson-Laird, P. N., \& Byrne, R. M. J. (2002). Conditionals: A theory of meaning, pragmatics, and inference. Psychological Review, 109, 646-678.

Kahneman, D., \& Miller, D. T. (1986). Norm theory: Comparing reality to its alternatives. Psychological Review, 93, 136-153.

Kahneman, D., \& TVersky, A. (1982). The simulation heuristic. In D. Kahneman, P. Slovic, \& A. Tversky (Eds.), Judgment under uncertainty: Heuristics and biases (pp. 201-208). New York: Cambridge University Press.

Kaschak, M. P., Madden, C. J., Therriault, D. J., Yaxley, R. H., Aveyard, M., Blanchard, A. A., \& Zwaan, R. A. (2005). Perception of motion affects language processing. Cognition, 94, B79-B89.

KaUP, B. (2001). Negation and its impact on the accessibility of text information. Memory \& Cognition, 29, 960-967.

KAUP, B., \& ZWAAN, R. (2003). Effects of negation and situational presence on the accessibility of text information. Journal of Experimental Psychology: Learning, Memory, \& Cognition, 29, 439-446.

LANGACKer, R. W. (1991). Foundations of cognitive grammar (Vol. 2). Stanford, CA: Stanford University Press.

LeSLIE, A. (1987). Pretense and representation: The origins of "theory of mind." Psychological Review, 94, 412-426.

MacDonald, M. C., \& Just, M. A. (1989). Changes in activation levels with negation. Journal of Experimental Psychology: Learning, Memory, \& Cognition, 15, 633-642.

McNamara, P., Durso, R., Brown, A., \& Lynch, A. (2003). Counter- factual cognitive deficit in persons with Parkinson's disease. Journal of Neurology, Neurosurgery, \& Psychiatry, 74, 1065-1070.

Münte, T. F., Schiltz, K., \& Kutas, M. (1998). When temporal terms belie conceptual order. Nature, 395, 71-73.

O’Brien, E. J., Cook, A. E., \& Peracchi, K. A. (2004). Updating situation models: Reply to Zwaan and Madden (2004). Journal of Experimental Psychology: Learning, Memory, \& Cognition, 30, 289-291.

Radvansky, G. A., Zacks, R. T., \& Hasher, L. (2005). Age and inhibition: The retrieval of situation models. Journals of Gerontology, 60B, P276-P278.

Roese, N. (1994). The functional basis of counterfactual thinking. Journal of Personality \& Social Psychology, 66, 805-818.

Roese, N. (1997). Counterfactual thinking. Psychological Bulletin, 121, 133-148.

RoESE, N. (2005). If only: How to turn regret into opportunity. New York: Broadway Books

Santamaría, C., Espino, O., \& Byrne, R. M. J. (2005). Counterfactual and semifactual conditionals prime alternative possibilities. Journal of Experimental Psychology: Learning, Memory, \& Cognition, 31, 1149-1154.

Thompson, V. A., \& Byrne, R. M. J. (2002). Reasoning counterfactually: Making inferences about things that didn't happen. Journal of Experimental Psychology: Learning, Memory, \& Cognition, 28, 1154-1170.

Urrutia, M., Moreno, V., de Vega, M., \& Morera, Y. (2005). Is counterfactual meaning grounded on perceptual/motor cognition? Symbols, Embodiment and Meaning: A debate. Garachico Workshop. Tenerife, Spain.

ZwaAn, R. A. (1996). Processing narrative time shifts. Journal of Experimental Psychology: Learning, Memory, \& Cognition, 22, 1196-1207.

ZwaAn, R. A., \& Madden, C. J. (2004). Updating situation models. Journal of Experimental Psychology: Learning, Memory, \& Cognition, 30, 283-288.

Zwaan, R. A., Madden, C. J., \& Whitten, S. N. (2000). The presence of an event in the narrated situation affects its availability to the comprehender. Memory \& Cognition, 28, 1022-1028.

\section{NOTE}

1. There are several counterfactual constructions. For instance, counterfactuals with the format "what might have been if . .." are useful to discover causal relations, whereas those of the type "if only ..." are associated with strong emotions. In this article, we are concerned only with conditional counterfactuals with explicit antecedent and consequents. 
APPENDIXA

Example of the Materials Translated From Spanish (Experiment 1)

Initial situation (always factual):

While keeping the ticket in her hand, / Mary switched on the radio / and heard the winning lottery number. /

Factual context:

As she won the lottery first prize, / the first thing she did /

Counterfactual context:

If she had won the lottery first prize, / the first thing she would have done /

Common subordinate clause:

was to buy a luxurious Mercedes car.

New situation final:

Critical clause: Mary got into the Mercedes car /

Postcritical clause: and she felt like a queen.

Old situation final:

Critical clause: Mary tore the ticket to pieces /

Postcritical clause: and started to clean the kitchen.

*** IS IT COHERENT? ${ }^{* * *}$

Probe:

${ }^{* * *} \operatorname{HEARD}^{* * *}$

APPENDIX B

Example of the Materials Translated From Spanish for Experiment 2

\section{Initial situation (always factual):}

John was still in the office / sitting in front of the computer. / He started to type a report / that his boss had asked him for. /

\section{Factual context:}

As he had enough time, / he went to the café / to drink a beer. /

\section{Counterfactual context:}

If he had had enough time, / he would have gone to the café / to drink a beer. /

\section{Probe:}

*** TYPE $^{* * *}$ (initial)

${ }^{* * *}$ DRINK $^{* * *}$ (final)

\section{Final sentence:}

John took his jacket off, / because the day was quite hot./

\section{Question:}

${ }^{* * *}$ Did the boss ask John for a report? ${ }^{* * *}$ 
APPENDIX C

Example of the Materials Translated From Spanish for Experiment 3

\section{Initial situation (always factual):}

John was still in the office / sitting in front of the computer. / He started to type a report / that his boss had asked him for. /

\section{Factual context:}

He went to the café / to drink a beer / because he had enough time. /

\section{Counterfactual context:}

He would have gone to the café / to drink a beer / if he had had enough time. /

\section{Final sentence:}

The day was quite hot. /

\section{Probe:}

*** TYPE ${ }^{* * *}$ (initial)

*** DRINK $^{* * *}$ (final)

Question:

${ }^{* * *}$ Did the boss ask John for a report? ${ }^{* * *}$

(Manuscript received March 29, 2006;

revision accepted for publication September 20, 2006.) 